\title{
Application of the fluorescence spectroscopy for the analysis of the state of abdominal cavity organs tissues in mini-invasive surgery
}

Ksenia Kandurova, Victor V. Dremin, Evgeny A. Zherebtsov, Elena V. Potapova, Andrey V. Dunaev, et al.

Ksenia Kandurova, Victor V. Dremin, Evgeny A. Zherebtsov, Elena V. Potapova, Andrey V. Dunaev, Andrian V. Mamoshin, Alexander L. Alyanov, Vadim F. Muradyan, "Application of the fluorescence spectroscopy for the analysis of the state of abdominal cavity organs tissues in mini-invasive surgery," Proc. SPIE 10685, Biophotonics: Photonic Solutions for Better Health Care VI, 106854P (17 May 2018); doi: 10.1117/12.2290037

SPIE. Event: SPIE Photonics Europe, 2018, Strasbourg, France 


\title{
Application of the fluorescence spectroscopy for the analysis of the state of abdominal cavity organs tissues in mini-invasive surgery
}

\author{
Ksenia Kandurova ${ }^{a^{*}}$, Viktor V. Dremin ${ }^{a}$, Evgeny A. Zherebtsov ${ }^{a, b}$, Elena V. Potapova ${ }^{a}$, \\ Andrey V. Dunaev ${ }^{\mathrm{a}}$, Andrian V. Mamoshin ${ }^{\mathrm{a}, \mathrm{c}}$, Alexander L. Alyanov ${ }^{\mathrm{a}, \mathrm{c}}$, Vadim F. Muradyan ${ }^{\mathrm{c}}$ \\ ${ }^{a}$ Orel State University named after I.S. Turgenev, Komsomolskaya St. 95, Orel, Russia, 302026; \\ ${ }^{\mathrm{b}}$ Optoelectronics and Biomedical Photonics Group, Aston Institute of Photonic Technologies, Aston \\ University, Aston Triangle, Birmingham, B4 7ET, UK; \\ ${ }^{\mathrm{c} O r e l}$ Regional Clinical Hospital, Orel, Russia, 302028
}

\begin{abstract}
At present, minimally invasive interventions become more widespread for treating hepatopancreatoduodenal area pathologies. However, new methods and approaches are necessary for obtaining more diagnostic information in real time. Several methods within the framework of "optical biopsy" concept are considered. The features and areas of application of each method are reviewed to find out which of them can be used in further studies to assess the possibility of intraoperative use in minimally invasive abdominal surgery. Preliminary measurements with fluorescence spectroscopy method have been performed at excitation wavelengths $365 \mathrm{~nm}$ and $450 \mathrm{~nm}$. Areas of interest were common bile duct, gallbladder and liver abscess. In our opinion, the obtained results can be a basis for further research and provide a deeper understanding of pathological processes of abdominal cavity organs tissues.
\end{abstract}

Keywords: optical diagnostics, optical biopsy, spectroscopy, imaging, fluorescence spectroscopy, minimally invasive surgery, hepatopancreatoduodenal area

\section{INTRODUCTION}

Currently, diseases of the hepatopancreatoduodenal area remain an urgent problem of abdominal surgery. Such pathologies as pancreatic cancer, gallstone disease and liver abscesses are steadily increasing incidence, complications probability and mortality rates ${ }^{1,2}$. Another problem is that in most cases patients are admitted to hospitals due to complications as early diagnosis rate does not increase.

In recent years, the interest in the applications of minimally invasive techniques for diagnosing and treating surgical pathology of abdominal organs has been increasing. Minimally invasive surgery allows one to decrease traumatism and scope of interventions, as well as the number of postoperative complications. Compared to conventional open surgery, minimally invasive surgery allows for patients to shorten hospital stay and recovery time ${ }^{3}$. However, one of the central problems of these techniques still requires being solved. It is the expansion of possibilities for diagnostic information intraoperative obtaining.

The most accurate method used to establish a diagnosis is a histological examination of samples obtained by biopsy or in an intraoperative way. This method is a gold standard, and its results can be used as a reference for other methods. However, development of minimally invasive technologies determines the need for new diagnostic methods allowing obtaining information comparable to these classical methods, but intraoperatively in vivo. Development and implementation of new diagnostic methods in clinical practice will improve the quality of treatment by increasing the level of reliability of diagnosis of the tissue state and optimizing algorithms of diagnostic and treatment.

Currently, the optical diagnostic methods are widely used in various areas of science, including medicine. One of the dynamically developing areas of application of these methods is determining the state of biological tissues during surgery. The methods used for the solution of this problem are often combined by the single term "optical biopsy" in literature. This field of research is quickly developing worldwide ${ }^{4}$. The advantage of these methods is a possibility to conduct research and obtain information in real time without the need to obtain biological material from the body, as in a

*kandkseniya@gmail.com; phone +7 910 2682946; http://www.bmecenter.ru/en 
routine biopsy. Optical biopsy methods are much various. They include fluorescence and diffuse reflectance spectroscopy, near infrared spectroscopy, Raman spectroscopy, confocal and two-photon laser endomicroscopy, optical coherence tomography, laser speckle contrast imaging, etc.

Fluorescence spectroscopy (FS) is one of the most popular optical spectroscopic methods for in vivo diagnostics. It is based on probing biological tissue with monochromatic optical radiation and recording excited fluorescence spectra. FS is an actively used method of studying the structure, biochemical properties of cells and tissues. The optical properties of fluorophores are sensitive to the metabolism conditions in biological tissues, which makes FS a valuable tool for studying the state of biological tissues (healthy, inflamed, malignant, etc.). ${ }^{5}$. This method allows one to record and analyze the emission of both administrated fluorescence contrast ${ }^{6}$ and endogenous substances that determine the metabolism and viability of cells and tissues in vivo ${ }^{7}$.

One of the applications of FS is assessing metabolic activity of tissues by studying the fluorescence intensity of nicotine adenine dinucleotide (NADH) and flavinoadenidine dinucleotide (FAD) which are the components of tricarbonic acid cycle. The reduced form of NADH coenzyme has a fluorescence intensity maximum at a wavelength of $490 \mathrm{~nm}$ when excited with $365 \mathrm{~nm}$ radiation. Oxidized FAD coenzyme has a maximum value of fluorescence intensity in the range of $520-540 \mathrm{~nm}$ when excited with $450 \mathrm{~nm}$ radiation ${ }^{8}$. The rate of accumulation of these coenzymes in mitochondria is related to the processes of oxidative phosphorylation. The intensity of metabolic processes can be estimated according to the content of NADH and FAD which affects fluorescence spectra.

Thus, FS has wide opportunities for early diagnosis of destructive inflammatory processes and monitoring of metabolic rate in biological tissues ${ }^{9}$. FS is used as an effective diagnosis method in many areas of medicine (for example, in the diagnosis of metabolic disorders in diabetes mellitus ${ }^{10}$ ), including surgery and oncology ${ }^{11,12}$.

Another widespread and effective non-invasive optical diagnostic method is diffuse reflectance spectroscopy (DRS). ${ }^{13}$ This method designed to evaluate absorbing and scattering properties of biological tissues. DRS is based on tissue illumination with broadband radiation. Diffuse reflectance spectra caused by multiple nonelastic scattering of light in tissue are detected. The study of oxygenation and blood perfusion of tissues using DRS is based on the difference in absorption spectra of oxyhemoglobin $(\mathrm{HbO} 2)$ and deoxyhemoglobin $(\mathrm{Hb})$. Reflected radiation provides information about tissue structure, perfusion of blood vessels, the spatial distribution of chromophores and their concentration ${ }^{14}$. Similar method - near-infrared spectroscopy - is used to evaluate blood oxygenation in studies of brain activity and for visualization in laparoscopic surgical interventions ${ }^{15}$.

Spectroscopy methods combined with conventional microscopy and endoscopy are another rapidly developing area of optical biopsy. These technologies are more complex than classical light microscopy, but open possibilities to perform real-time histologic and cytological biopsies ${ }^{16}$ in vivo. Confocal and two-photon laser endomicroscopy allow studying individual fluorophores molecules, observing dynamic processes in cells at various depths and reconstructing a threedimensional image of them.

Therefore, an optical biopsy is actively developing breakthrough direction, which includes a large number of different methods, characterized by different complexity and application areas. Joint application of several techniques as part of the multidimensional approach provides the most valuable information for diagnosis and treatment. However, their application in the field of minimally invasive surgery is associated with a number of technological and methodological challenges at the present time.

To make preliminary studies, FS was chosen as a relatively simple and affordable, but still sensitive method of optical diagnostics. The purpose of this work was to evaluate the possibilities of using FS method for intraoperative recording of fluorescence spectra and obtaining information about the metabolic activity of cells and state of tissues and mucous membranes in areas of the pathology in hepatopancreatoduodenal organs.

\section{THE METHOD OF RESEARCH}

Experimental measurements were performed using specially developed fiber optic system with a laparoscopic optical probe. The main units of the system were designed in cooperation with SPE "LAZMA" Ltd (Moscow, Russia). Radiation sources with wavelengths of $365 \mathrm{~nm}$ and $450 \mathrm{~nm}$ were used for endogenous fluorescence excitation. Fluorescence spectra were registered with a spectrometer in the range of 350-820 nm. 
24 patients with abdominal organs pathologies of Orel Regional Clinical Hospital (Orel, Russia) surgery department aged $66 \pm 10$ years participated in preliminary experimental studies conducted as an additional measurement procedure in the frame of prescribed minimally invasive surgical interventions. The studies were approved by Ethics committee of Orel State University named after I.S. Turgenev (record of the meeting №10 of 16.11.2017). Patients signed an informed consent indicating their voluntary willingness to participate in the study.

Patients were divided into 3 groups depending on the area of interest and clinical task: proximal common bile duct (14 patients with obstructive jaundice caused by pancreatic cancer or cholelithiasis), the body of the gallbladder (5 patients with acute destructive cholecystitis) and the wall of liver abscess (4 patients). The experimental measurements were conducted during minimally invasive diagnostic and therapeutic interventions under ultrasonic and X-ray examination. Areas of interest are shown in Figure 1.

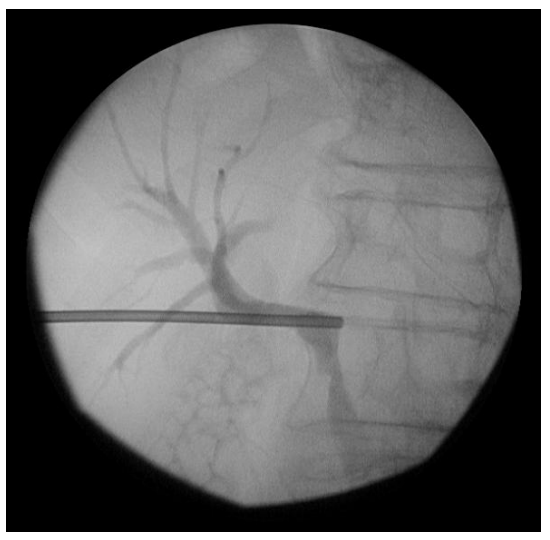

(a)

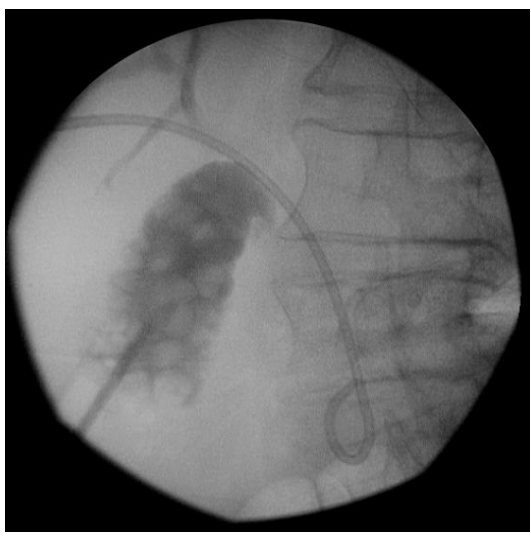

(b)

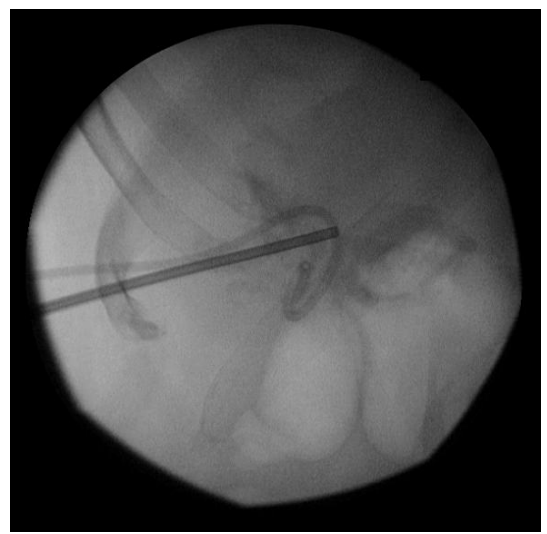

(c)

Figure 1. The areas of interest: common bile duct (a), gallbladder (b), liver abscess.

Spectra were registered three times in each area at each wavelength and then were averaged for further processing. In order to correctly compare the data obtained from different points, obtained fluorescence intensities were normalized using the following formula:

$$
I_{\text {norm }}(\lambda)=\frac{I(\lambda)}{I_{\max \text { source }}}
$$

$I(\lambda)$ is fluorescence intensity at $\lambda \mathrm{nm}$ wavelength, $I$ is the maximum intensity of backscattered radiation. This formula allows compensating significant scatter between raw fluorescence intensities in observed spectra.

\section{RESULTS AND DISCUSSION}

The processing of obtained data showed that the fluorescence intensity and backscattered radiation intensity is a highly variable parameter. Significant scatter of raw data was observed. For example, fluorescence intensities at a wavelength of $365 \mathrm{~nm}$ lie within 300-600 units for the common bile duct, 300-900 units for the gallbladder and 100-300 units for an abscess. For a wavelength of $450 \mathrm{~nm}$, the intensities ranged from 150-250 units, 150-500 units and 50-100 units respectively. This fact caused the use of normalization formula 1.

Figure 2 shows the average normalized fluorescence spectra of the proximal part of the common bile duct, gallbladder body and liver abscess wall at excitation wavelength $365 \mathrm{~nm}$. 


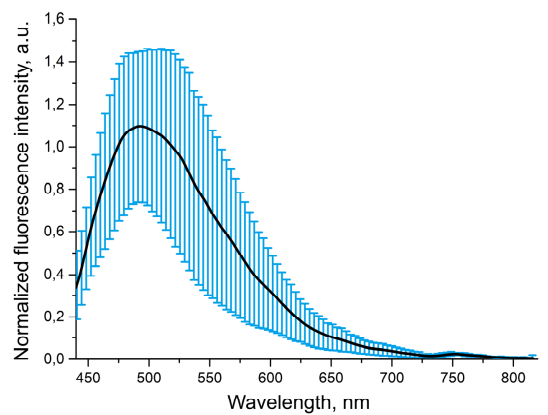

(a)

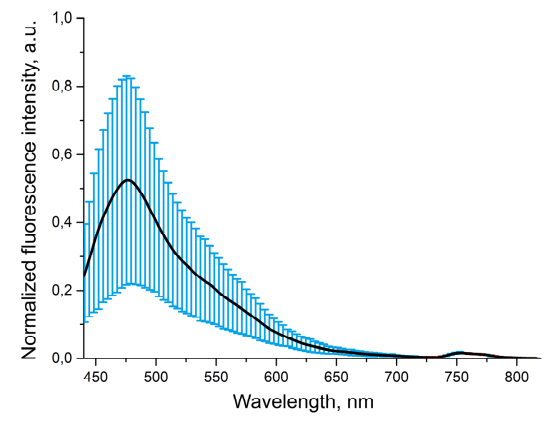

(b)

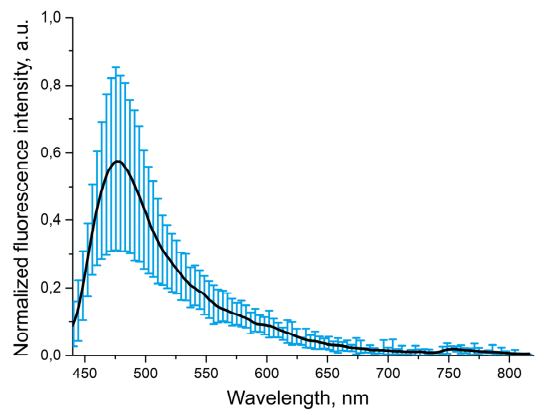

(c)

Figure 2. Average fluorescence spectra of the common bile duct (a), gallbladder (b) and liver abscess at excitation wavelength $365 \mathrm{~nm}$.

In return, Figure 3 shows the average normalized fluorescence spectra for areas of interest at excitation wavelength 450 $\mathrm{nm}$.

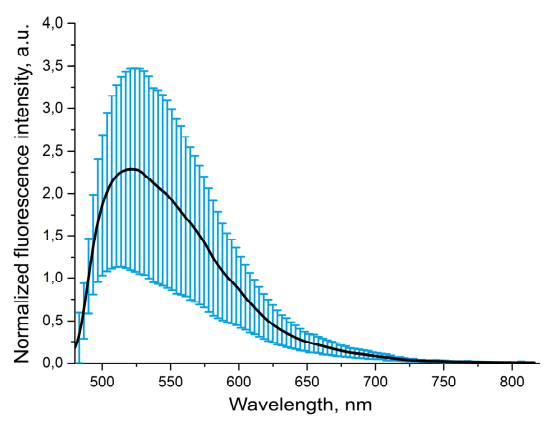

(a)

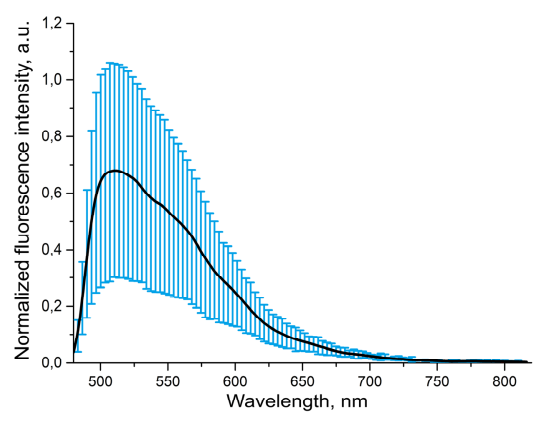

(b)

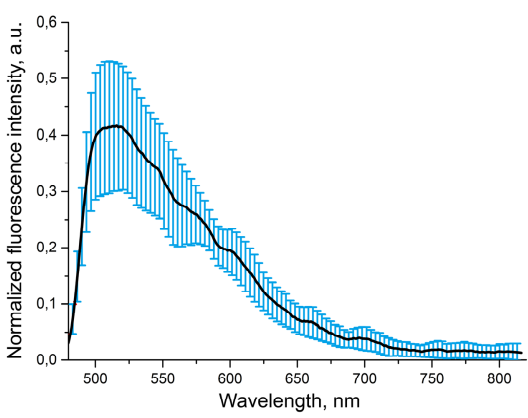

(c)

Figure 3. Average fluorescence spectra of the common bile duct (a), gallbladder (b) and liver abscess at excitation wavelength $450 \mathrm{~nm}$.

In several cases influence of blood perfusion of tissues was noted in the form of decreases in fluorescence intensity at wavelengths around $540 \mathrm{~nm}$ and $580 \mathrm{~nm}$ associated with the absorption of laser radiation by oxyhemoglobin $\left(\mathrm{HbO}_{2}\right)^{17}$. Blood is a good absorber of optical radiation in visible and ultraviolet ranges, which leads to signal weakening and further misinterpretation of the results. ${ }^{18}$ It is supposed that of a number of other factors also has a significant influence on obtained data.

\section{CONCLUSION}

Conducted studies have shown the significant potential of FS method in minimally invasive surgery as well as further development of this modality for the purposes of optical biopsy.

The results and obtained preliminary data demonstrated the sensitivity of this method to the properties of the area of interest and kind of pathological changes. However, it is assumed that the obtained spectra are influenced by a number of various factors, such as the state and type of changes in tissues, the phase development of pathological processes, their response to current treatment, the filling of blood with tissue, the presence of other liquids, etc. There is a need to study the contribution of each factor in tissue fluorescence for correct interpretation of diagnostic data.

The next step in conducting research using fluorescence spectroscopy is the development and application of the multimodal approach to the analysis and interpretation of the registering data, which will be based on the diffuse reflectance and laser speckle contrast measurements of the organs under study. 
In addition, it is impossible to speak about the robustness of the obtained results without comparison with the fluorescence spectra of the tissues of the organs under study in the absence of pathology. It should be noted, that the specificity of the study did not allow us to obtain a control group and to obtain diagnostic data on healthy volunteers in vivo. In the near future, the comparative measurements in affected and nonaffected regions will be conducted in vivo. The overall aim of the measurements will be the development of diagnostic criteria to increase the effectiveness of treatment procedures in the hepatopancreatoduodenal zone in the frame of the mini-invasive surgical interventions.

\section{ACKNOWLEDGEMENTS}

This study was supported by the Russian Science Foundation under project № 18-15-00201.

\section{REFERENCES}

[1] Ilic, M. and Ilic, I., "Epidemiology of pancreatic cancer," World Journal of Gastroenterology 22(44), 9694-9705 (2016).

[2] Shaffer, E.A. "Epidemiology and risk factors for gallstone disease: Has the paradigm changed in the 21st century?" Current Gastroenterology Reports 7(2), 132-140 (2005).

[3] Jelínek, F., Arkenbout, E.A., Sakes, A. and Breedveld, P., "Minimally invasive surgical instruments with an accessory channel capable of integrating fibre-optic cable for optical biopsy: A review of the state of the art," Proceedings of the Institution of Mechanical Engineers, Part H: Journal of Engineering in Medicine 228(8), 843-853 (2014).

[4] Kennedy, G.T., Okusanya, O.T., Keating, J.J., Heitjan, D.F., Deshpande, C, Litzky, L.A., et al., "The optical biopsy: A novel technique for rapid intraoperative diagnosis of primary pulmonary adenocarcinomas," Annals of Surgery 262(4), 602-609 (2015).

[5] Dremin, V.V. and Dunaev, A.V., "How the melanin concentration in the skin affects the fluorescencespectroscopy signal formation," Journal of Optical Technology 83(1), 43-48 (2016).

[6] Stelmashchuk, O., Zherebtsov, E., Zherebtsova, A., Kuznetsova, E., Vinokurov, A., Dunaev, A., Mamoshin, A., Snimshchikova, I., Borsukov, A., et al., "Noninvasive control of the transport function of fluorescent coloured liposomal nanoparticles," Laser Physics Letters 14(6), (2017).

[7] Dremin, V.V., Margaryants, N.B., Volkov, M.V., Zhukova, E.V., Zherebtsov, E.A., Dunaev, A.V., and Rafailov, E.U., "Assessment of tissue ischemia of nail fold precapillary zones using a fluorescence capillaroscopy," Proc. SPIE 10412, Diffuse Optical Spectroscopy and Imaging VI, 104120W (2017).

[8] Bartolomé, F. and Abramov, A.Y., "Measurement of mitochondrial NADH and FAD autofluorescence in live cells," Methods in .Molecular Biology 263-270 (2015).

[9] Mayevsky, A., [Mitochondrial function in vivo evaluated by NADH fluorescence], Springer International Publishing, (2015).

[10] Dremin, V.V., Zherebtsov, E.A., Sidorov, V.V., Krupatkin, A.I., Makovik, I.N., Zherebtsova, A.I., et al.. "Multimodal optical measurement for study of lower limb tissue viability in patients with diabetes mellitus," Journal of Biomedical Optics 22(8), (2017).

[11] Bulgakova, N., Ulijanov, R., Vereschagin, K., Sokolov, V., Teplov, A., Rusakov, I., et al., "In vivo local fluorescence spectroscopy in PDD of superficial bladder cancer," Medical Laser Application 24(4), 247-255 (2009).

[12] Rafailov, I.E., Dremin, V.V., Litvinova, K.S., Dunaev, A.V., Sokolovski, S.G. and Rafailov, E.U., "Computational model of bladder tissue based on its measured optical properties," Journal of Biomedical Optics 21(2), 025006 (2016).

[13]Zherebtsov, E., Popov, A., Doronin, A., Meglinski, I., and Bykov, A., "Hyperspectral system for Imaging of skin chromophores and blood oxygenation," Proc. SPIE 10412, Diffuse Optical Spectroscopy and Imaging VI, 104120G (2017).

[14] Potapova, E.V., Dremin, V.V., Zherebtsov, E.A., Makovik, I.N., Zherebtsova, A.I., Dunaev, A.V., et al., "Evaluation of microcirculatory disturbances in patients with rheumatic diseases by the method of diffuse reflectance spectroscopy," Human Physiology 43(2), 222-228 (2017). 
[15]Zarrinpar,. A, Dutson, E.P., Mobley, C., Busuttil, R.W., Lewis, C.E., Tillou, A., et al., "Intraoperative Laparoscopic Near-Infrared Fluorescence Cholangiography to Facilitate Anatomical Identification: When to Give Indocyanine Green and How Much,” Surgical Innovation. 23(4), 360-365 (2016).

[16] Liu, J., Dlugosz, A. and Neumann, H., "Beyond white light endoscopy: The role of optical biopsy in inflammatory bowel disease," World Journal of Gastroenterology 19(43), 7544-7551 (2013).

[17] Zherebtsov, E.A., Kandurova, K.Y., Seryogina, E.S., Kozlov, I.O., Dremin, V.V., Zherebtsova, A.I., et al., "The influence of local pressure on evaluation parameters of skin blood perfusion and fluorescence," Progress in Biomedical Optics and Imaging - Proceedings of SPIE 10336,1033608 (2017).

[18]Zherebtsov, E., Dremin, V., Zharkikh, E., Zherebtsova, A., Rafailov, I., Dunaev, A., Bazieva, N., Rafailov, E., Science, A., et al., "Fibre-optic probe for fluorescence diagnostics with blood influence compensation" Proc. SPIE 10493, Dynamics and Fluctuations in Biomedical Photonics XV, 104931L, 1-6 (2018). 